\title{
HRTEM Observation and Atomic Modeling of $\alpha / \beta$ Interphase Boundary in a Ti-22V-4Al Alloy
}

\author{
Naoki Miyano $^{1}$, Kei Ameyama ${ }^{2}$ and George C. Weatherly ${ }^{3}$ \\ ${ }^{1}$ Research Organization of Science and Engineering, Ritsumeikan University, Kusatsu 525-8577, Japan \\ ${ }^{2}$ Department of Science and Engineering, Ritsumeikan University, Kusatsu 525-8577, Japan \\ ${ }^{3}$ Department of Materials Science and Engineering, McMaster University, Hamilton, L8S 4M1, Canada
}

\begin{abstract}
In order to study interface boundary structures, a three dimensional Near-Coincidence Site lattice (Near-CSL) model based on Bollmann's O-lattice method has been proposed. Previous studies have shown that intragranular $\alpha$-phase precipitates in a Ti-22V-4Al alloy have a morphology of lath-like, with two well defined facet planes. A detailed TEM observation of the laths has been made in this study and the results correlated to the present analysis. Accommodation mechanism of misfit dislocations and the Burgers vector of the defects observed at the broad face and side facet plane of the intragranular $\alpha$-phase precipitates have been determined. The structural ledges at the side facet, as predicted by the present analysis, is consistent with HRTEM observation. The Burgers vector of misfit dislocations at the broad face is in good agreement with that previously reported for a $\mathrm{Zr}-2.6 \mathrm{Nb}$ alloy, that has almost the same lattice parameter ratio as the Ti-22 $\mathrm{V}-4 \mathrm{Al}$ alloy used in the present study. Although the Burgers vector of the defects on the broad face can be determined by HRTEM observation, the boundary plane is not well defined. However, it can be demonstrated that the interface boundary structure of the broad face can be rationalized from the results of both the Near-CSL analysis and the HRTEM observations.
\end{abstract}

(Received December 3, 2001; Accepted April 5, 2002)

Keywords: interphase boundary structure, misfit dislocation, structural ledge, Burgers vector, near-coincidence site lattice, precipitate, orientation relationship, invariant line

\section{Introduction}

The morphology of intragranular precipitates formed as a result of a diffusional phase transformation depends on the crystallographic orientation relationship (O.R.) and interphase boundary structure between the precipitate and matrix phase. To study the O.R. and the interphase boundary structure, geometrical approaches, e.g. those based on the O-lattice, ${ }^{1)} \mathrm{CSL}^{2)}$ or the invariant line ${ }^{3)}$ model, are correlated with experimental observations. In a previous study, ${ }^{4}$ a three-dimensional near coincidence site lattice (Near-CSL) model, ${ }^{4-10)}$ based on the CSL model ${ }^{2)}$ which investigates an atomic matching, has been developed to explain the experimental observations of intragranular $\alpha$ precipitates (HCP) in a Ti-22V-4Al alloy obtained by transmission electron microscope (TEM). The $\alpha$ precipitate has a morphology of lathlike, bounded by three planes: two prominent facets are the side facet and broad face. Although structural ledges were observed at the side facet of the $\alpha$ precipitates the Burgers vector of the ledges was not determined. On the broad face, the misfit dislocations were observed to be paired, ${ }^{4)}$ although why pairing of dislocation exists or the mechanism of misfitaccommodation at the broad face was not elucidated.

In the present study, we have studied the $\alpha / \beta$ interphase boundary structures in more detail, focusing on the structure of the broad face of the $\alpha$ precipitates. The Burgers vectors of both the structural ledges at the side facet and the misfit dislocations at the broad face are determined by combining the HRTEM results with the Near-CSL model.

\section{Experimental Procedure and Modeling}

A Ti-22V-4Al alloy containing $\mathrm{V}: 22.2, \mathrm{Al}: 4.2, \mathrm{Fe}: 0.14$, O: $0.11, \mathrm{~N}: 0.008, \mathrm{C}: 0.008$ and H: 0.017 (mass\%) was pre- pared by vacuum melting. The $\beta$ transus of the material is approximately $1000 \mathrm{~K}$. Therefore, the alloy was solution treated at $1573 \mathrm{~K}$ for $18 \mathrm{ks}$ and subsequently aged at $923 \mathrm{~K}$ for $400 \mathrm{ks}$ under a $\mathrm{He}$ gas atmosphere, followed by furnace cooling to obtain an $(\alpha+\beta)$ microstructure. TEM samples were prepared by ion milling and examined in a JEOL 2010F highresolution TEM at $200 \mathrm{kV}$. The relative orientation relationship of $\alpha$ precipitates and $\beta$ grains was determined by Kikuchi pattern analysis. The error associated with this technique has been assessed from the analysis of annealing twins in FCC crystals. This gave an average error of less than $0.10^{\circ}$ in the orientation relationships. ${ }^{11)}$

In the Near-CSL analysis, the calculated procedure for determining the coincident atoms is as follows: the lattices of the matrix phase and the precipitate phase are overlapped in a three-dimensional space with a specific O.R. Then, the coincident atoms are identified from the overlapped atoms of matrix and precipitate when the relative distance between two atoms is less than some critical value, $d_{\max }$ which is a purely geometrical value. The distribution and ratio of the coincident atoms are then employed to examine the inter-dependence of the O.R. and interphase boundary structures.

The distribution and density of the coincidence atoms depends strongly on the criterion chosen for $d_{\max }$. A smaller $d_{\max }$ value indicates a more sensitive and accurate distribution of the coincidence atoms. However, it may lead to a less accurate representation of the experimental results which often include a small misorientation in the O.R. Therefore, a $d_{\max }$ value of $15 \%$ for the nearest-neighbor distance has been employed in our previous work. ${ }^{4-6)}$ Comparing the Near-CSL results using $d_{\max }=15 \%$ with O-lattice theory in a FCC/BCC two phase mixture leads to good agreement with the observations. ${ }^{5)}$ 


\section{Results}

\subsection{Experimental observations}

Figure 1 shows a SEM back scattered image of a specimen annealed at $923 \mathrm{~K}$ for $400 \mathrm{ks}$. As seen in this figure, the intragranular $\alpha$ phase has a lath shape. A schematic illustration of the complete interphase structure of an $\alpha$ lath in a $\beta$ matrix is shown in Fig. 2, as discussed in our previous work. ${ }^{4)}$ The apparent boundary of the broad face is approximately $(6 \overline{7} 6)_{\beta}$. A periodic array of misfit dislocations lying parallel to the lattice invariant line, $\sim\left[\begin{array}{lll}43 & 69 & 37\end{array}\right]_{\beta}$ in this alloy, is present at the broad face. The apparent boundary plane of the side facet is approximately $\left(\begin{array}{lll}17 & 3 & 14\end{array}\right)_{\beta}$ and consists of structural ledges. The interphase structure of each facet, as determined by HRTEM, is shown in the following figures.

Figure 3 shows a high-resolution transmission electron micrograph of the interphase structure of the side facet. The side facet boundary contains an array of structural ledges about $9 \mathrm{~nm}$ in width and $2.5 \mathrm{~nm}$ in height, while the terrace plane is parallel to $(0001)_{\alpha} /(\overline{101})_{\beta}$. The broad face of an $\alpha$ lath is shown in Figs. 4(a) and (b). Figure 4(a) illustrates the structure of the broad face at a near edge-on orientation with $g=0002$. As shown in this figure, the array of misfit dislocations consists of paired sets of closely spaced dislocations separated by individual dislocations at a slightly greater spacing. Figure 4(b) is a high-resolution transmission electron micrograph of the boxed area indicated in Fig. 4(a). Extra-half

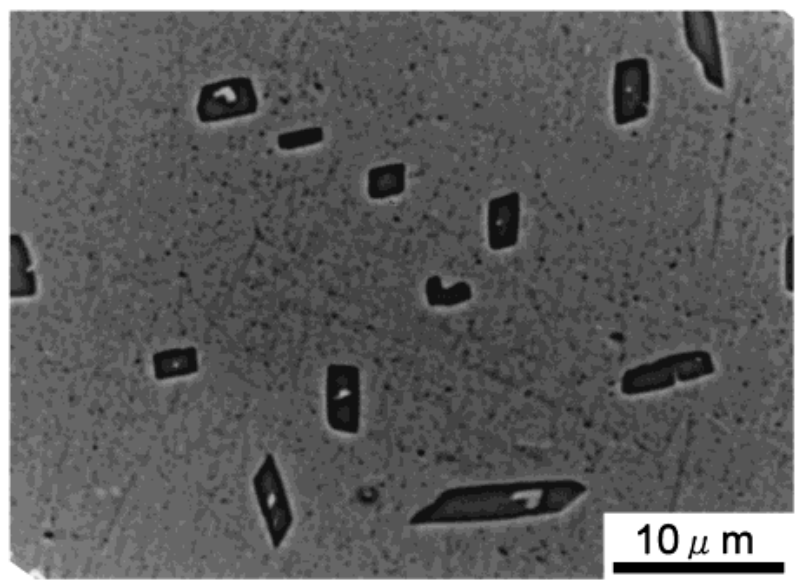

Fig. 1 SEM image of a specimen aged at $923 \mathrm{~K}$ for $400 \mathrm{ks}$. An $\alpha$ lath precipitate in $\beta$ grain.

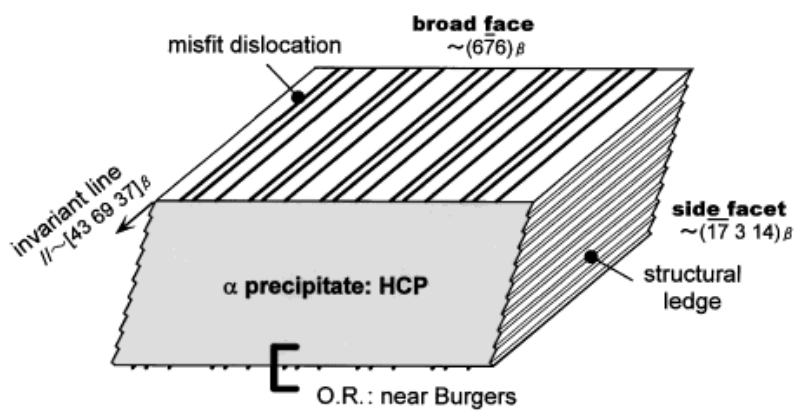

$\beta$ matrix: BCC

Fig. 2 A schematic illustration of the interphase structures of an $\alpha(\mathrm{HCP})$ lath in $\beta$ (BCC) matrix. planes can be seen in the image, marked by the arrows in the figure. It should be noted that the dislocations marked as "A" and "B" lie on the same $(01 \overline{1} 1)_{\alpha} /(\overline{1} 10)_{\beta}$ plane. This suggests that the broad face of the precipitate contains structural steps, although the nature of these steps cannot be determined from this figure. Figure 5 shows Inverse Fast Fourier Transform (IFFT) images of the square areas surrounding each of the dislocations A, B and C seen in Fig. 4(b). The numbers in these figures represent the number of layers associated with each plane. The image of each dislocation is associated with two extra-half planes, one with the $(0001)_{\alpha} /(\overline{101})_{\beta}$ set of planes and the other with the $(0 \overline{1} 11)_{\alpha} /(0 \overline{1} 1)_{\beta}$ set. The three dislocations have identical Burgers vectors.

\subsection{Modeling results and discussion}

To apply the Near-CSL model to the present alloy, BCC and HCP lattices having the same lattice parameter ratio as the alloy $\left(a_{\beta}=0.3206, a_{\alpha}=0.2938\right.$ and $\left.c_{\alpha}=0.4712[\mathrm{~nm}]\right)$ are fixed in a specific O.R., as determined by a Kikuchi pattern analysis. In this alloy the $\alpha$ precipitates have a nearBurgers O.R. ${ }^{12)}$ with the $\beta$ matrix. The coordinates of the near-coincident atoms are determined from the analysis, and their positions can then be depicted in a three-dimensional plot. Figure 6 shows the distribution of the coincident atoms and the trace of the apparent habit plane of the broad face and side facet, as observed by TEM. The coincident atom "clusters" are arranged regularly and line-up along the lattice in-

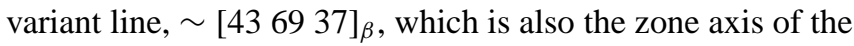
two facets. Hence, the structure of each facet can be investigated by viewing the plots of the near coincident atoms along the invariant line direction, as seen in the following figures.

\subsubsection{Side facet}

Figure 7 shows the distribution of coincident atom clusters at the side facet, as viewed along the lattice invariant line direction. The apparent habit plane of the side facet is $\sim\left(\begin{array}{lll}\overline{17} & 3 & 14\end{array}\right)_{\beta}$. Using the hypothesis that planes with a high density of coincidence atoms correspond to lower energy boundaries, the solid line in this figure is the prediction of the "best-matching" interphase boundary. ${ }^{5)}$ The predicted interphase boundary has regular structural ledges, $9 \mathrm{~nm}$ in width, $2.5 \mathrm{~nm}$ in height with the terrace plane parallel to $(0001)_{\alpha} /(\overline{101})_{\beta}$. This is in excellent agreement with the structure of the interphase boundary plane as observed in Fig. 3. Figure 8 shows an image of a structural ledge on the side facet determined from the Near-CSL analysis, where the positions of all the $\alpha$ and $\beta$ atoms, as seen in the projection along the invariant line, are plotted. A Burgers circuit can be constructed, see Fig. 8, to determine the component of the displacement associated with the step lying in the plane of the projection. From the circuit, the component of the Burgers vector of the structural ledge in this projection is determined to be parallel to a direction defined by

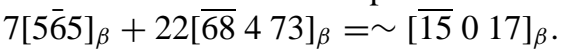

\subsubsection{Broad face}

The morphology of $\alpha$ precipitates in a $\beta$ grain in a $\mathrm{Zr}-$ 2.6 mass $\% \mathrm{Nb}$ alloy, that has almost the same lattice parameter ratio as the $\mathrm{Ti}-22 \mathrm{~V}-4 \mathrm{Al}$ alloy, is similar to that observed in this alloy. ${ }^{13)}$ In the $\mathrm{Zr}-\mathrm{Nb}$ study, it was reported that the Burgers vector of the misfit dislocations on the broad face is $\langle 001\rangle_{\beta}$. Therefore, it can be assumed that the Burgers vector 


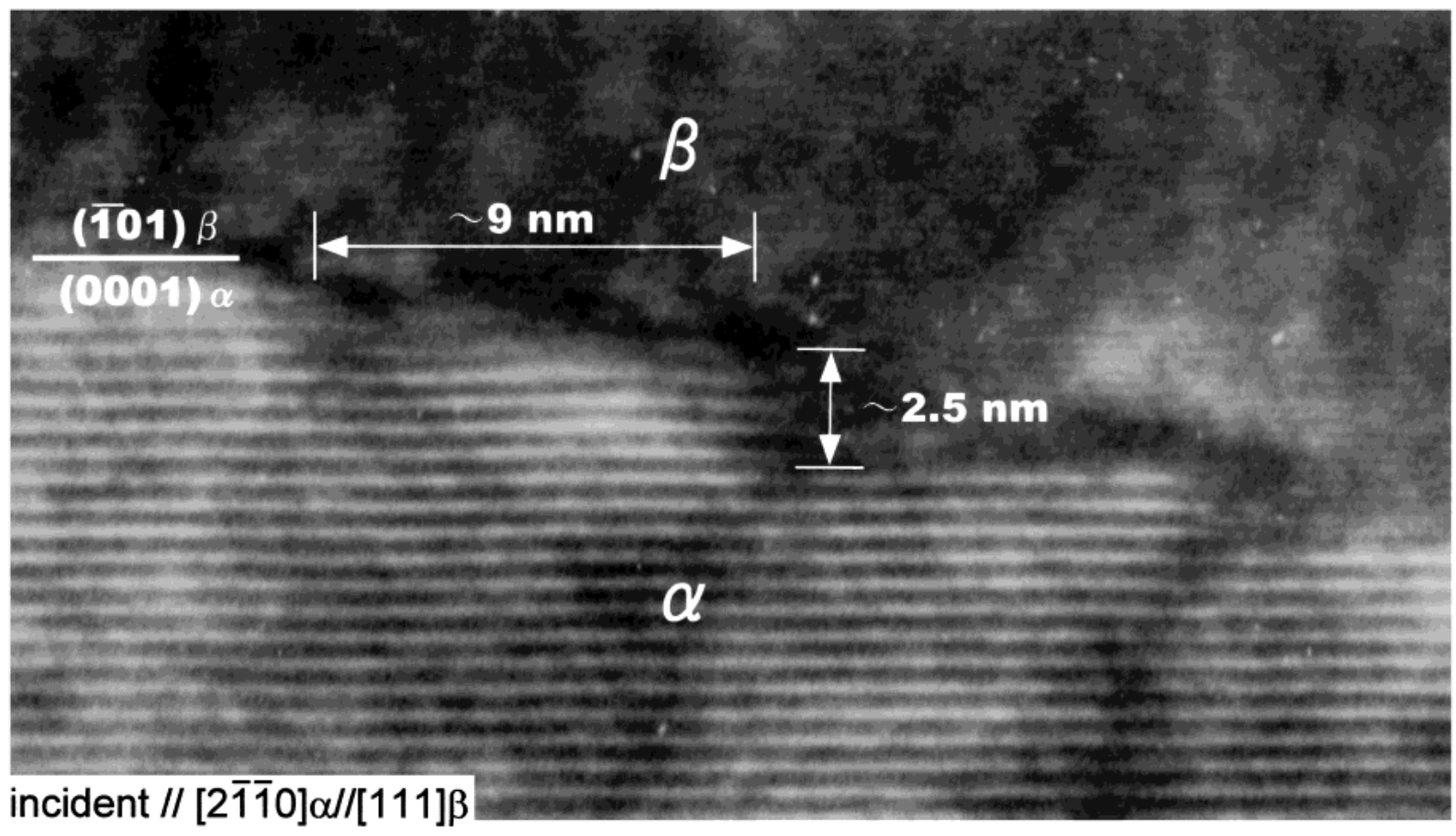

Fig. 3 A high resolution image of $\alpha / \beta$ interphase boundary structure at the side facet of an $\alpha$ lath.
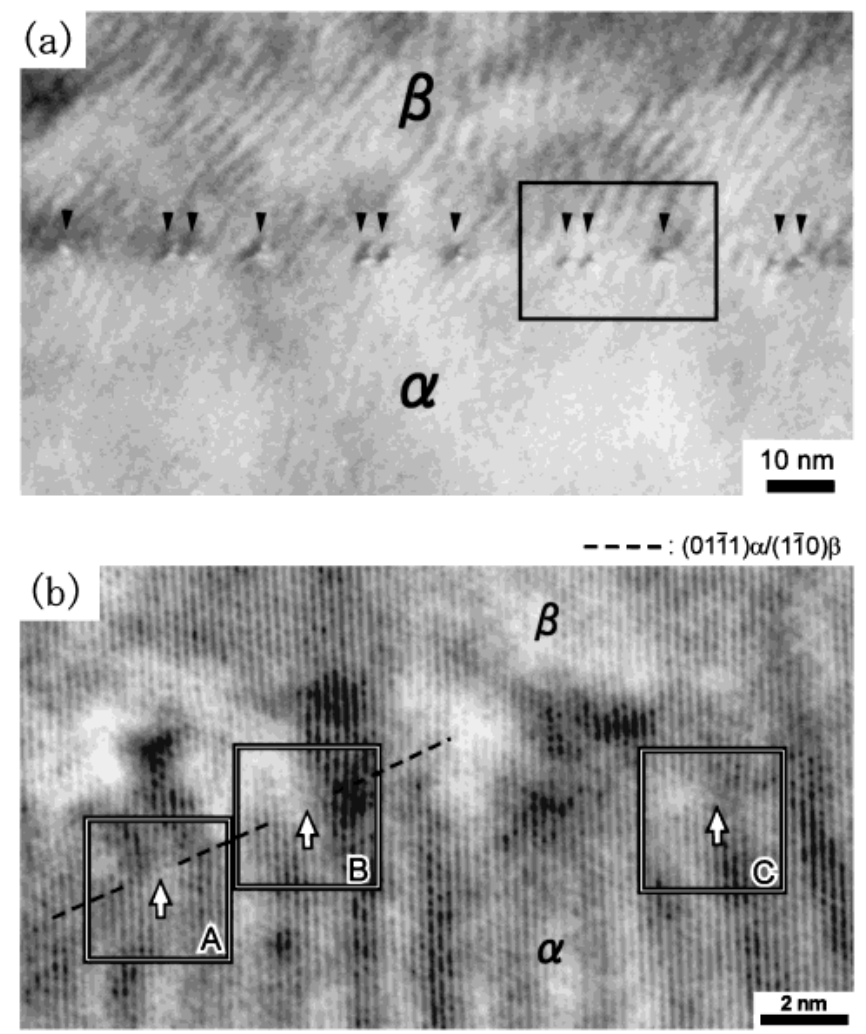

Fig. $4 \alpha / \beta$ interphase boundary structure of the broad facet of an $\alpha$ lath, (a) at near-edge on $(g=0002)$, (b) a high resolution image of the squared area in Fig. 4(a).

in this Ti22 $\mathrm{V} 4 \mathrm{Al}$ alloy is also $\langle 001\rangle_{\beta}$. If the Burgers vector is $[001]_{\beta}$, an extra-half plane would be seen in the HRTEM image associated with the $(0001)_{\alpha} /(\overline{1} 01)_{\beta}$ and $(0 \overline{1} 11)_{\alpha} /(0 \overline{1} 1)_{\beta}$ sets of planes, but not in the $(01 \overline{1} 1)_{\alpha} /(\overline{1} 10)_{\beta}$ set. The reason for this observation is obvious when we check the inner prod-

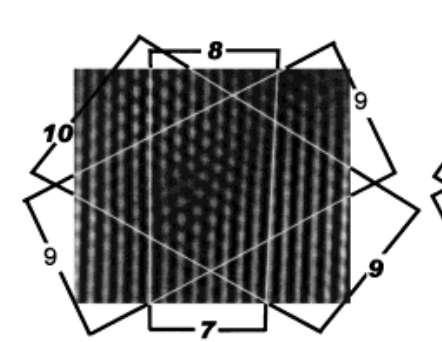

A

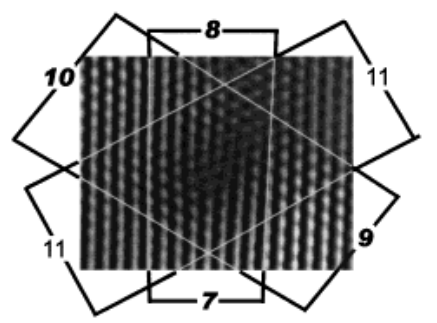

C

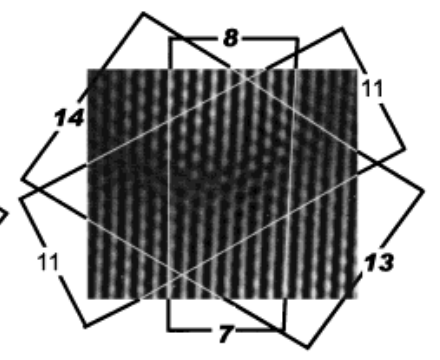

B

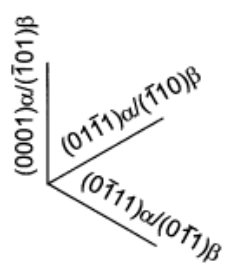

Fig. 5 IFFT images of the squared areas surrounding each of the dislocations A, B and C seen in Fig. 4(b).

uct of $[001]_{\beta}$ with $[\overline{1} 01]_{\beta},[0 \overline{1} 1]_{\beta}$ and $[\overline{1} 10]_{\beta}$ respectively; only the product of $[001]_{\beta}$ with $[\overline{1} 10]_{\beta}$ is zero, i.e. the Burgers vector has no component in the $(01 \overline{1} 1)_{\alpha} /(\overline{1} 10)_{\beta}$ plane. This result is in full agreement with the experimental observations shown in Fig. 5; i.e. the Burgers vector of the dislocation on the broad face is $[001]_{\beta}$.

Although there is no clear evidence for the formation of structural ledges at this interphase from the HRTEM images, the positions of the dislocations seen in Fig. 4 suggest that structural ledges might be present. Hence, the NearCSL analysis and experimental results have been compared in terms of misfit accommodation at the broad face, in or- 


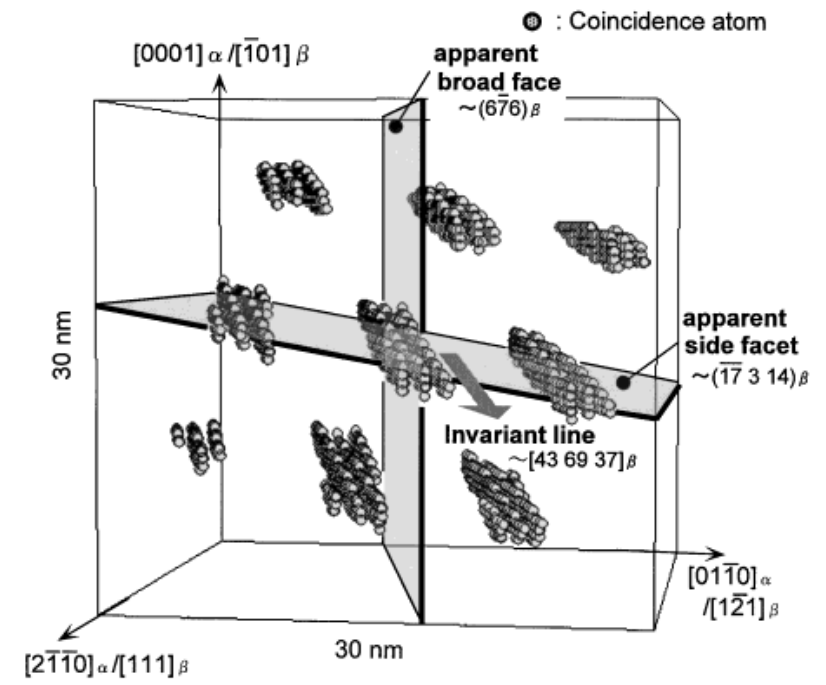

Fig. 6 Distribution of the coincidence atoms in a 3-D space. The O.R. between BCC and HCP lattice is a near-Burgers O.R.

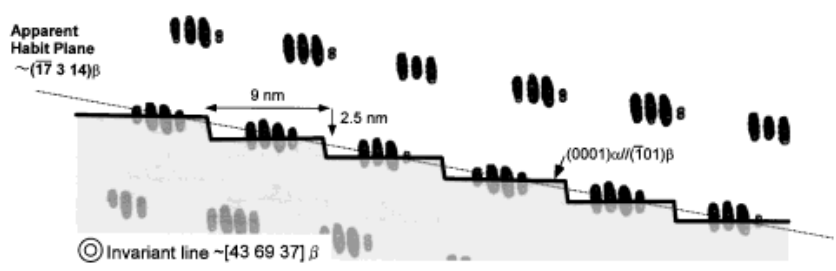

Fig. 7 The coincidence atom clusters and the $\alpha / \beta$ interphase boundary structure of the side facet, which is predicted by the present Near-CSL analysis.

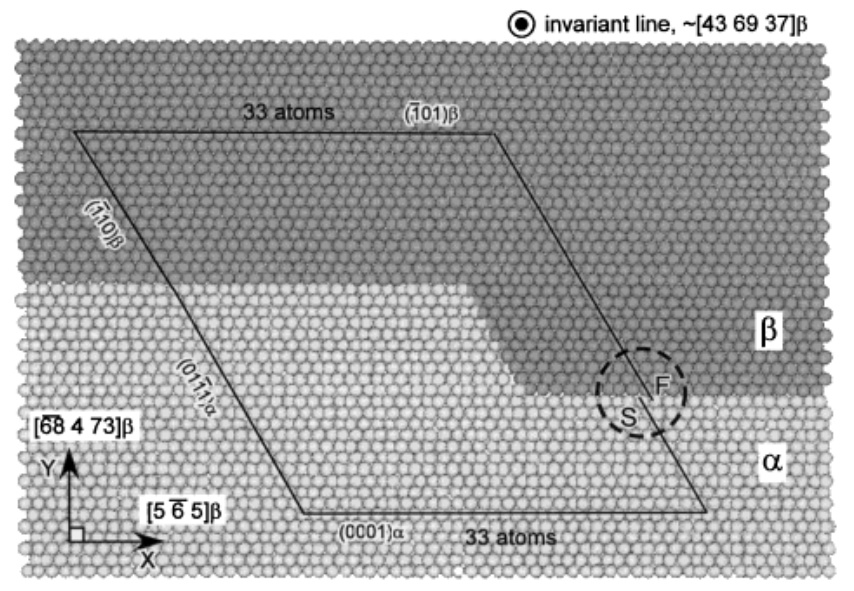

Fig. 8 Atomic image and Burgers circuit (FS/RH) of the structural ledge on the side facet by Near-CSL analysis, viewing along invariant line direction $\sim\left[\begin{array}{lll}43 & 69 & 37\end{array}\right]_{\beta}$.

der to investigate whether facets might be energetically favored at the broad face. Figure 9(a) shows the distribution of coincident atom clusters viewed along the lattice invariant line direction. The apparent habit plane of the broad face is $\sim(6 \overline{7} 6)_{\beta}$. The accommodation of the misfit at the apparent habit plane must account for the misfit between the $(0001)_{\alpha} /(\overline{101})_{\beta}$ set of planes, because the broad face is almost perpendicular to $(0001)_{\alpha} /(\overline{1} 01)_{\beta}$. The average spacing of the dislocations calculated by the difference between the $\mathrm{d}$-spacings of the $(0001)_{\alpha} /\{110\}_{\beta}$ planes is $\sim 8 \mathrm{~nm}$. The experimental results also give $8 \mathrm{~nm}$ as the average dislocation

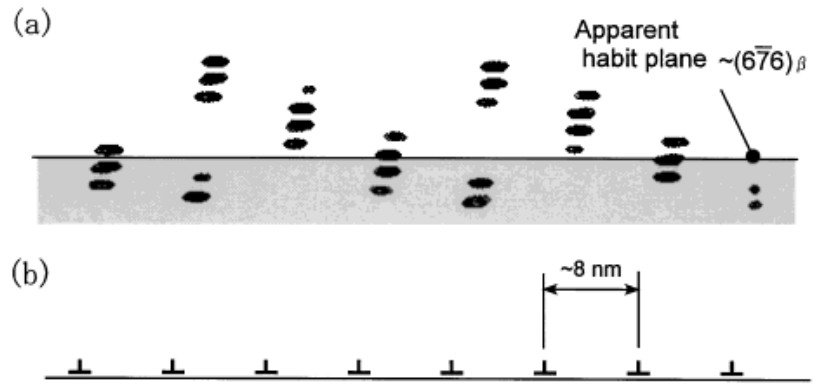

(c)

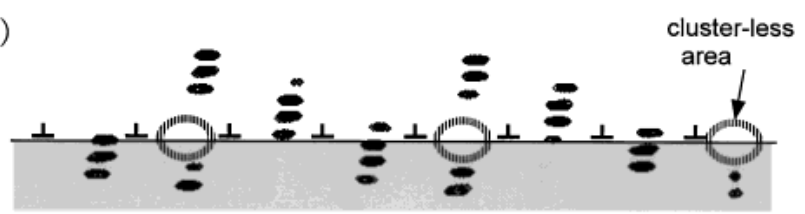

(d)

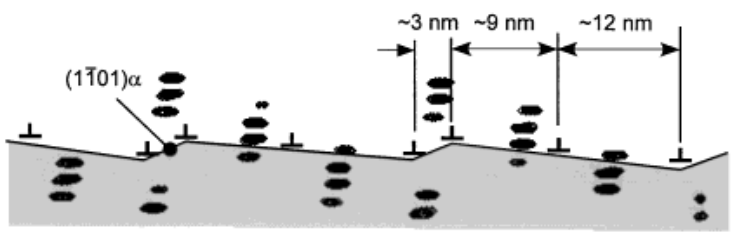

Fig. 9 (a) The distributions of the coincidence atom clusters and the apparent habit plane of the broad face. (b) A arrangement of the misfit dislocations calculated by misfit between the plane space of $(0001)_{\alpha}$ and $\{110\}_{\beta}$ on the broad face. (c) The resulting distribution of coincidence atoms and the set of misfit dislocations produced by overlapping the images of (a) and (b). (d) One possibility of a low-energy boundary structure, as a result of shifting of the space and position of dislocations.

spacing (see Fig. 4(a)). Therefore, the dislocations indicated by the symbol $\perp$ can be first introduced to lie precisely on the apparent habit plane, as shown in Fig. 9(b). Figure 9(c) shows the resulting distribution of coincidence atoms and the set of misfit dislocations produced by overlapping the images of Figs. 9(a) and (b). If we focus on the distribution of the clusters of coincidence atoms on the apparent broad face in Fig. 9(c), we can note that "cluster-less" areas (between the dislocations) appear periodically, $24 \mathrm{~nm}$ apart, as seen in the figure. If the "cluster-less" areas are regarded as patches which have a (local) higher interfacial energy than the other areas, this could provide an explanation for the pairing of the dislocations. The high-energy of the "cluster-less" areas might provide a local driving force for shifting the spacing and position of the dislocations, in order to reduce the total energy of the boundary. One possibility for how this might be achieved is shown in Fig. 9(d). After shifting the position of the dislocations, the "cluster-less" areas are now confined to the low-index plane $\{1 \overline{1} 01\}_{\alpha}$, lying between the paired dislocations, while each facet between the paired dislocations has a cluster of coincidence atoms corresponding to a low-energy plane. The total energy of the broad face is thereby reduced.

\section{Conclusions}

The aim of this study was to make detailed TEM observations and apply a Near-CSL analysis in order to understand the mechanism of misfit accommodation at the broad and side faces of intragranular $\alpha$ precipitates in a Ti-22V-4Al alloy.

The Near-CSL analysis is able to predict the $\alpha / \beta$ inter- 
phase boundary structure of the side facet. The predicted boundary consists of low index planes, and shows good correspondence with the experimental results.

The Burgers vector of dislocations found at the broad face is $[001]_{\beta}$. However the distribution of these dislocations is not uniform, and HRTEM studies show that pairing of the dislocations is observed on the $\{1 \overline{1} 01\}$ plane. This pairing is thought to be related to the reduction in the interfacial energy that can be achieved by allowing the apparent habit plane to facet, such that the faceted boundary passes through regions of highest coincidence, as determined from the NearCSL analysis.

\section{REFERENCES}

1) W. Bollmann: Phlys. Status Solidi (a) 21 (1974) 543-550.
2) P. H. Pumphrey: Grain Boundary Structure and Properties, ed. by G. A. Chadwick and D. A. Smith (Academic Press, N. Y., 1976) pp. 139-200.

3) U. Dahmen: Acta Metall. 30 (1982) 63-73.

4) N. Miyano, K. Ameyama and G. C. Weatherly: ISIJ Inter., Supplement 40 (2000) S199-S203.

5) N. Miyano and K. Ameyama: J. Japan Inst. Metals 64 (2000) 42-49.

6) N. Miyano, K. Ameyama and G. C. Weatherly: Mater. Sci. Eng. A, (in press).

7) Q. Liang and W. T. Reynolds, Jr.: Metall. Mater. Trans. A 29A (1998) 2059-2072.

8) J. M. Rigsbee and H. I. Aaronson: Acta Metall. 27 (1979) 351-363.

9) M. G. Hall, H. I. Aaronson and K. R. Kinsman: Surf. Sci. 31 (1972) 257-274.

10) T. Furuhara and H. I. Aaronson: Acta Metall. 39 (1991) 2857-2872.

11) K. Ameyama, M. Yanagida and M. Tokizane: J. Japan Inst. Metals 57 (1993) 726-731.

12) W. G. Burgers: Physica 1 (1934) 561-586.

13) W.-Z. Zhang, V. Perovic, A. Perovic, G. C. Weatherly and G. R. Purdy: Acta Metall. 46 (1998) 3443-3453. 